\title{
Patientenverfügung: ethische Erwägungen zum neuen Erwachsenenschutzrecht unter besonderer Berücksichtigung der Demenz
}

Susanne Brauer

\section{Der Artikel beruht auf der NEK-CNE Stellungnahme Nr.17/2011 und übernimmt teilweise Textauszüge. Die Seitenzahlen beziehen sich auf den Text der Stellung- nahme. Diese ist in Deutsch, Französisch, Italienisch und Englisch unter www.nek-cne.ch verfügbar oder als Broschüre zu beziehen.}

Korrespondenz: Susanne Brauer, PhD Geschäftsstelle Nationale Ethikkommission im Bereich Humanmedizin

c/o Bundesamt für Gesundheit Seilerstrasse 8

CH-3003 Bern

susanne.brauer@bag.admin.ch
Am 1. Januar 2013 wird das neue Erwachsenenschutzrecht (EWS) in Kraft treten. Damit werden sich medizinische Entscheidungsprozesse für nicht mehr urteilsfähige Patientinnen und Patienten bundesweit nachhaltig ändern, wenn eine Patientenverfügung (PV) verfasst wurde. Die PV übernimmt die wichtige Funktion, verbindlich den Willen einer Person auszudrücken, die aufgrund ihrer Urteilsunfähigkeit einen solchen nicht mehr bilden kann. Ziel ist es, den Patientinnen und Patienten in der Schweiz damit einen neuen Raum der Selbstbestimmung zu eröffnen.

Die Nationale Ethikkommission im Bereich Humanmedizin begrüsst die rechtliche Neuerung. Zugleich erschien es der Kommission erforderlich, eine vertiefte ethische Reflexion über die Voraussetzungen für die Gültigkeit einer PV und die Grenzen ihrer Verbindlichkeit bei der Umsetzung zu führen. Besonders strittig sind diese Punkte mit Blick auf Demenz. Entsprechend konzentrierte sich die Kommission in ihrer Meinungsbildung auf entsprechende Problematiken, wohlwissend, dass diese auch die PV im Allgemeinen, unabhängig von einem bestimmten Krankheitstyp, betreffen.

\section{Selbstbestimmung stärken braucht auch Fürsorge}

Für die Kommission steht unbestritten fest: Die PV ist Ausdruck eines Abwehrrechts gegen medizinische Eingriffe in die (körperliche und psychische) Integrität einer Person, das im ethischen Prinzip der Patientenautonomie gründet und anzuerkennen ist. Es ist jedoch nicht mit einem Anspruchsrecht auf Leistungen, die medizinisch und pflegerisch nicht notwendig sind, zu verwechseln. Ein solches Anspruchsrecht besteht nicht.

Es würde dem Grundgedanken der Autonomie widersprechen, wenn der Entscheid, eine PV zu verfassen, nicht freiwillig geschehen würde, indem beispielsweise das Vorliegen einer PV Bedingung für die Aufnahme in eine Behandlungs- und Pflegeinstitution sei. In den Augen der Kommission ist darauf zu achten, dass diese Freiwilligkeit stets gegeben ist.

Dass der Mensch in der Ausübung seiner Autonomie auf andere Menschen und deren Fürsorge angewiesen ist, wird besonders bei der PV deutlich (vgl. S. 17). Denn ob sein Wille auch im Zustand seiner Urteilsun- fähigkeit tatsächlich umgesetzt wird, hängt allein von anderen Menschen ab. Sie sind dafür verantwortlich, die in der PV formulierten Anordnungen zu situationsbezogenen Anweisungen zu konkretisieren und entsprechend zu handeln, um so der betroffenen Person zu einer selbstbestimmten Behandlung zu verhelfen. Eine sorgfältige, dem Willen der betroffenen Person gerecht werdende, situationsbezogene Umsetzung der PV ist als eine Pflicht der Fürsorge zu verstehen, deren Verletzung Folgen für das therapeutische Vertrauensverhältnis hätte. Denn eine solche Verletzung würde ein falsches Signal setzen, nämlich dass über verletzbare Personen, die sich aufgrund ihrer Urteilsunfähigkeit nicht mehr gegen medizinische Eingriffe zur Wehr setzen können, Drittpersonen nach eigenen Vorstellungen vom Guten und Richtigen verfügen dürften.

\section{Aus einer Demenz-Diagnose darf nicht automatisch auf eine Urteils- unfähigkeit geschlossen werden.}

Das Prinzip der Fürsorge greift auch noch in weiterer Hinsicht. So trägt die Ärzteschaft (und nach dem neuen Erwachsenenschutzrecht auch die vertretungsberechtigte Person) weiterhin Verantwortung für die Behandlung und Betreuung urteilsunfähiger Personen. Mit der PV wird allerdings eine Gewichtung der Perspektive vorgenommen, unter der die medizinische oder pflegerische Entscheidung zu treffen ist: nämlich vorrangig aus der Perspektive der betroffenen Person, wie sie in der PV formuliert ist. Das entbindet aber die Personen, die die Entscheidung ausführen, nicht von den oben erwähnten Fürsorgepflichten.

In diesem Sinn versteht die Kommission die Fürsorge als Ergänzung zur Patientenautonomie, um den Bereich der Selbstbestimmung durch die PV effektiv $\mathrm{zu}$ vergrössern.

\section{Ohne das geht es nicht: Grundvoraussetzungen der PV}

Aus Sicht der Ethik betont die Kommission drei Grundvoraussetzungen, die erfüllt sein müssen, da- 


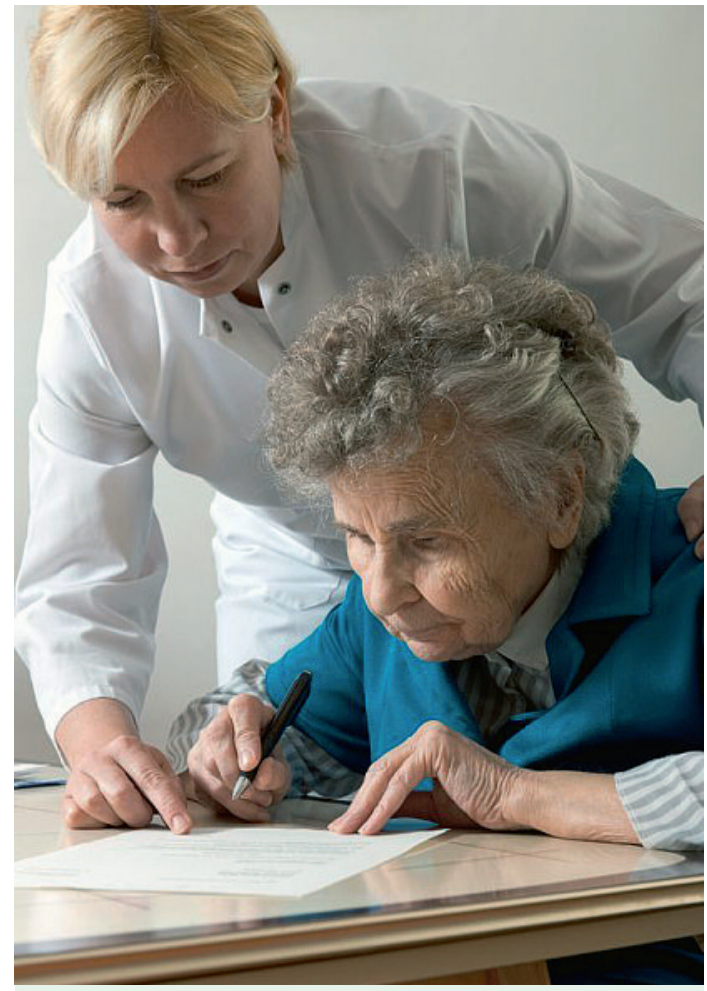

Nur eine urteilsfähige Person kann eine rechtsgültige Patientenverfügung verfassen.

mit die PV eine verbindliche Willensäusserung ist. Alle drei Voraussetzungen sind nach Meinung der Kommission auch bei Demenz erfüllt (vgl. S. 6f.).

- Die Kommission folgt dem Erwachsenenschutzrecht, das von einer «Kontinuität der Person» ausgeht. Das heisst, die Umsetzung einer PV betrifft die Person, die auch die PV verfasst hat. Diese Beobachtung ist nicht trivial und wird im Zusammenhang mit Demenz teilweise in Zweifel gezogen. Diese Zweifel teilt die Kommission nicht.
Demenz ist es wichtig, für die Beurteilung die «guten Zeiten» der Person abzupassen. Zudem sollte schriftlich festgehalten werden, wer nach welchen Kriterien und mit welchen Methoden bzw. auf welche Art und Weise die Abklärung von Urteilsfähigkeit vorgenommen hat, weil ein einheitlicher Standard für das Verfahren in der Medizin bislang fehlt.

- Um eine PV verfassen zu können, muss der Mensch in der Lage sein, sich zukünftige Krankheitszustände vorzustellen. Nur dann kann er einen antizipierten Willen bilden. Die Kommission geht davon aus, dass Urteilsfähigkeit die Fähigkeit zur Antizipation auch mit Blick auf Demenz einschliesst. Von einer Beratungspflicht und der Forderung, dass die Person die Gründe für ihre Entscheidung sichtbar machen muss, sieht die Kommission ab. Entscheidend bleibt für die Kommission die Freiheit, auch sogenannte «unvernünftige» Entscheide treffen zu dürfen.

\section{Wo die Verbindlichkeit an ihre Grenzen stösst}

Auch für die Kommission gibt es Bereiche, über die man nicht verbindlich verfügen kann. Sie stellt sich damit auf den Standpunkt des neuen Erwachsenenschutzrechts sowie bestehender nationaler und kantonaler Gesetze und folgt der Position der SAMW. Einschränkungen des Gegenstandsbereichs bleiben für die Kommission jedoch begründungsbedürftig. Mit Blick auf Einschränkungen bezüglich pflegerischer und schmerzbekämpfender Massnahmen führt die Kommission Gründe der Sozialethik, der beruflichen Integrität und der Fremdschädigung an. Aus diesen Überlegungen ergeben sich für die Kommission folgende Konsequenzen (vgl. S. 7 u. 25):

- Ein Angebot von patientengewohnter Nahrung, Körperpflege, Bewegung und Beschäftigung ist stets zu erbringen. Eine PV darf nicht verfügen, ein solches Angebot zu unterlassen.

\section{Künstliche Ernährung als ein akutmedizinischer Eingriff hingegen darf in einer Patientenverfügung verbindlich abgelehnt werden.}

- Die Urteilsfähigkeit ist für die Gültigkeit der PV entscheidend: Nur eine urteilsfähige Person kann eine rechtsgültige PV verfassen; und diese darf nur dann zur Entscheidungsgrundlage werden, wenn die Person urteilsunfähig geworden ist. Der Kommission ist es wichtig zu betonen: Aus einer Demenz-Diagnose darf nicht automatisch auf eine Urteilsunfähigkeit geschlossen werden. Urteilsunfähigkeit ist bezogen auf eine konkrete Situation immer sorgfältig abzuklären. Angesichts fluktuierender Zustände von Urteilsfähigkeit bei
- Massnahmen für die «Aktivitäten des täglichen Lebens» dürfen jedoch nicht mit Zwang durchgesetzt werden. Ein Mensch mit Demenz hat immer noch das Recht, beispielsweise Nahrung zu verweigern. Es ist jedoch zu prüfen, ob organische Ursachen oder irrationale Ängste für das Patientenverhalten vorliegen, die dann zunächst behoben werden müssten.

- Künstliche Ernährung als ein akutmedizinischer Eingriff hingegen darf in einer PV verbindlich abgelehnt werden. 
- Die Verabreichung von Medikamenten darf in einer PV verbindlich abgelehnt werden, es sei denn, sie dient dazu, unerträgliche Schmerzen zu lindern oder psychische Ursachen eines selbstschädigenden Verhaltens zu beheben.

\section{Knackpunkt: das Verhältnis zwischen mut- masslichem Willen und PV}

Die PV wird im neuen Erwachsenenschutzrecht behandelt wie eine aktuelle Willensäusserung. Darin besteht ihre Verbindlichkeit, die von der Kommission begrüsst wird. Ihre Gültigkeit kann jedoch nach dem Erwachsenenschutzrecht in Zweifel gezogen werden, wenn sie nachweislich nicht mehr dem mutmasslichen Willen* der betroffenen Person entspricht. Die Kommission steht diesem Vorgehen ambivalent gegenüber, auch wenn sie ebenfalls an der Möglichkeit der Korrektur und des Widerrufs einer PV festhalten will. Auf der einen Seite befürchtet sie Unsicherheiten seitens der Ärzteschaft bezüglich der Verbindlichkeit von PV. Durch die Einführung des mutmasslichen Willens könnte eine Kultur des Zweifels entstehen, die zu einer routinemässigen Überprüfung der PV führen könnte und sie damit praktisch in ihrer Verbindlichkeit herabsetzen würde. Dabei ist zu bedenken, dass der mutmassliche Wille eine Konstruktion von Drittpersonen ist, die vor allem auch ihre Sicht auf die betroffene Person widerspiegelt. Bei der Konstruktion sind daher besondere Sorgfaltskriterien zu beachten (s. u.). Auf der anderen Seite sollten nach Meinung der Kommission die im Erwachsenenschutzrecht vorgesehen Möglichkeiten des Widerrufs einer PV erweitert werden, d. h. eine PV sollte auch mündlich widerrufen werden können.

\section{Gesichtspunkte bei der Ermittlung des mutmasslichen Willens}

Um eine Fremdbestimmung der urteilsunfähigen Person zu vermeiden, schlägt die Kommission besondere Sorgfaltskriterien für die Ermittlung des mutmasslichen Willens vor (vgl. S. 8 u. 29).

Die Kommission geht davon aus, dass

- nur Personen ermitteln, die der Patientin bzw. dem Patienten wohlwollend gegenüberstehen;

- eigene Interessen zugunsten des Patientenwohls oder -willens zurückgestellt werden und der eigene Werthorizont und die eigenen Einstellungen bei der Ermittlung mitreflektiert werden, um Entscheidungen im Sinne der Person zu treffen;

- den ermittelnden Personen bewusst ist, dass der mutmassliche Wille vom objektiven Patientenwohl abweichen kann.

Zum gründlichen Vorgehen bei der Ermittlung des mutmasslichen Willens gehören:

- Mündliche Aussagen, welche die Person gegenüber Ärztin und Arzt macht, werden im Patientendossier dokumentiert. Mündliche Aussagen ausserhalb der Arzt-Patienten-Beziehung müssen von mehreren Personen bezeugt werden können.

- Der Grad der Demenz wird professionell und sorgfältig abgeklärt, um sicherzustellen, dass die mündliche Äusserung zeitlich in eine Phase gefallen ist, in der die betroffene Person urteilsfähig war.

\section{Aktuelle Gewohnheiten und Bedürfnisse hebeln eine PV nicht aus}

Gerade mit Blick auf Demenz wird oft die Frage aufgeworfen, ob aktuell geäusserte Gewohnheiten und Bedürfnisse einer urteilsunfähigen Person eine PV revidieren dürfen. Das Erwachsenenschutzrecht schliesst diese Möglichkeit aus, weil der mutmassliche Wille nur auf Äusserungen einer urteilsfähigen Person basiert. Auch in den Augen der Kommission wäre es nicht rechtens, eine PV in ihrer Verbindlichkeit anzuzweifeln mit dem Verweis auf Gesten, Reaktionen oder verändertes Verhalten der urteilsunfähigen Person. Solche Gewohnheiten und Bedürfnisse auch als eine veränderte Einstellung gegenüber medizinischen Belangen auszulegen, beruht allein auf der Vorstellung und den Rückschlüssen von Drittpersonen und widerspricht letztlich der Einschätzung einer Urteilsunfähigkeit: Denn eine urteilsunfähige Person ist per definitionem nicht mehr in der Lage, Entscheidungen über medizinische Belange zu treffen, weil ihr ein Verständnis über den Inhalt, die Tragweite und mögliche Folgen der Entscheidung gänzlich fehlt. Dieser Umstand schliesst jedoch nicht aus, Äusserungen einer urteilsunfähigen Person im Betreuungsalltag zu berücksichtigen - jedoch nur mit Blick auf die Lebensbereiche auf die sich die Äusserungen tatsächlich beziehen.

\section{Ziele der fürsorgerischen Unterbringung im Blick behalten}

Für die Gültigkeit einer PV spielt es aus ethischer Sicht keine Rolle, an welcher Krankheit die Person leidet. Dennoch kann es bei psychischen Krankheiten, zu denen im neuen Erwachsenenschutzrecht auch die Demenz zählt, zu einer Situation kommen, in denen die Verbindlichkeit einer Verfügung relativiert wird, nämlich innerhalb einer fürsorgerischen Freiheitsentziehung (neu: «fürsorgerische Unterbringung»). Im Namen des fürsorgerischen Schutzes wird das Abwehrrecht von psychisch erkrankten Personen eingeschränkt. Die Kommission stimmt dem Vorgehen $\mathrm{zu}$, soweit Fremdgefährdung oder schwere Verwahrlosung, die Grund für eine «fürsorgerische Unterbringung» sind, vorliegen oder Bereiche betroffen sind, über die ein Mensch nicht verbindlich verfügen darf. Die Kommission plädiert jedoch für ein verantwortungsvolles Abwägen zwischen Forderungen der Fürsorge und der in der Regel prioritären Selbstbestimmung. Entscheidungen sollten hier unter Einbezug des Behandlungs- und Betreuungsteams, der vertretungsberechtigten Person und einer möglichen Vertrauensperson breit abgestützt werden. Die Kom- kann nach Schweizer Recht von einer urteilsfähigen Person gebildet werden. 
mission bekräftigt aber, dass Anweisungen einer gültigen PV, die nicht die Gründe der «fürsorgerischen Unterbringung» betreffen, weiterhin befolgt werden müssen, z. B. die Anweisung, nicht reanimiert zu werden.

\section{Stellvertreterentscheide als Auftakt zu einem} gemeinsamen Entscheidungsmodell

Nach neuem Erwachsenenschutzrecht soll die behandelnde Ärztin bzw. der behandelnde Arzt unter Einbezug der vertretungsberechtigten Person medizinische Entscheidungen für urteilsunfähige Personen treffen, wenn keine PV vorliegt. Das Gesetz sieht eine genaue Abfolge von vertretungsberechtigten Personen vor. Eine PV kann auch dazu genutzt werden, eine vertretungsberechtigte Person zu bestimmen. zur Patientin bzw. zum Patienten überfordern könnte. Für die Ermittlung des mutmasslichen Willens, für die die Person verantwortlich ist, sind die oben angeführten Sorgfaltskriterien zu berücksichtigten.

Problematisch erscheint es der Kommission, dass laut neuem Erwachsenenschutzrecht sich die Entscheidungsträgerinnen und -träger nicht nur am mutmasslichen Willen orientieren sollen, sondern auch an den objektiven Interessen der erkrankten Person, d. h. an dem Patientenwohl, das sich nach medizinischen Kriterien definiert. Diese Konstellation kann zu Konflikten führen, weil der mutmassliche Willen keinen Vernünftigkeitskriterien standhalten muss, während die objektiven Interessen gerade an solche gebunden sind. Die Kommission empfiehlt daher eine klare Rangfolge der Entscheidungskrite-

\section{Problematisch: Laut neuem Erwachsenenschutzrecht soll sich der Entscheidungsträger nicht nur am mutmasslichen Willen orientieren, sondern auch an den objektiven Interessen der erkrankten Person.}

Die Kommission begrüsst den Wechsel zu einer gemeinsamen Entscheidungskompetenz zwischen Ärzteschaft und vertretungsberechtigter Person, die in der Regel Familienangehörige oder andere nahestehende Personen sind. Sie macht jedoch auch darauf aufmerksam, dass hier hohe Anforderungen an die vertretungsberechtigte Person gestellt werden, die sie unter Umständen nicht zuletzt aufgrund ihrer Nähe rien einzuhalten: Liegt keine PV vor, ist allein nach dem mutmasslichen Willen zu entscheiden. Erst wenn auch dieser nicht ermittelt werden kann, dürfen objektive Interessen für die Entscheidungsfindung herangezogen werden. Mit diesem Verfahren liessen sich die Selbstbestimmung von Patientinnen und Patienten und ihr Schutz vor Fremdbestimmung auch bei einer Demenz maximal sichern. 